\title{
Impact of Dynamic Thermal Conductivity Change of EPS Insulation on Temperature Variation through a Wall Assembly
}

\author{
Maatouk Khoukhi \\ College of Engineering, United Arab Emirates University \\ PO Box 15551, Al Ain, United Arab Emirates \\ mkhoukhi@uaeu.ac.ae; Tel.: +971-56950-6883
}

\begin{abstract}
This paper evaluates the effect of dynamic thermal conductivity $(\lambda)$ change of EPS insulation on temperature change through a conventional wall assembly at varying positions of the insulation within the assembly in question. According to the findings, in the case of the application of the variable $\lambda$-value of the insulation, compared to that obtained when the constant $\lambda$-value for polystyrene (EPS) insulation is adopted in the same conditions, the temperature profile through the wall assembly during the daytime is greater. In the event of applying the constant and variable $\lambda$ - values, the temperature shift on the inside is seen to decline as the location of the insulation material is positioned towards the surface of the inner wall. These results suggest that considering betterment in the insulation's thermal conductivity would provide the best dynamic thermal efficiency by placing the material in the middle of the wall assembly, taking into account the change in the thermal conductivity of the insulation.
\end{abstract}

Keywords: heat transfer; net thermal effect; dynamic thermal change; insulation material; variable thermal conductivity

\section{Introduction}

Global demand for energy is predicted to increase in the coming decades. In this context, buildings tend to consume energy at various levels at each point of the life cycle. In the field of construction, nearly $50 \%$ of non-renewable resources (such as water, electricity, and similar raw materials) consumed by humans are being put to use. For its continued survival, contemporary human society relies on buildings and what they contain; despite this, the existing level consumption linked to them will not sustain our planet [1]. Construction has also been seen to have significant effect on not only energy consumption but on how it translates into its impact on climate. For instance, a large share of this consumption is occupied by building materials. The vast majority of the products used use significant quantities of energy for transport [2]. The building envelope signifies elements like the wall, floor, window, ceiling, and door that isolate the building (conditioned space) from the outside and allow energy to be transferred inside or outside. It has a critical effect on energy consumption as an indoor and outdoor reagent [3]. While the cost of developing a building envelope is 15-40 percent of the total construction cost, its contribution to life-cycle costs, especially energy costs, is around 60 percent [4]. The building skin plays the role of a filter for regulating the intake of air, heat, cold and light between indoor and outdoor conditions [5]. The temperature changes in winter and summer can be reduced through the help of building envelopes. The material for thermal insulation acts as an important boundary; it thermally resists heat transfer into buildings by providing a barrier of any external environmental effects [6]. An insulation material provides high thermal resistance by declining the heat flow rate [7] and prevents external conditions from affecting its specific thermophysical properties [8]. The overall heat transfer coefficient (U-value) must be gauged when evaluating the energy performance of the building itself; this serves as the main function of thermal conductivity and in related calculations, it is also deemed a constant. As several works note, thermal conductivity acts as a function of different factors, which mainly include moisture content, temperature, and density, therefore this is an oversimplification.

Many works around the subject have been developed in the last decades [9-11]. These works found that the $\lambda$-value of materials for thermal insulation is directly proportional to temperature of the operation; the latter also increases when the former increases $[12,13]$. Several studies examining the change in the $\lambda$ values as a function of density, humidity, and operating temperature on the cooling capacity needed by buildings have recently been published [14, 15].The effect of moisture content on the thermal conductivity of the insulation material has also been studied by several authors [16-19]. 
Although the influence of surface temperature and humidity fluctuations and their effects on the change in the $\lambda$ value have been reported in previous works, the effect of the insulation material's location within the wall section was not studied intensively to select the optimum less-thermal dynamically effected position. The key aim of this study is to highlight the dynamic thermal performance of the insulation material at various locations of the wall section. Given the aforementioned observations, the main objective of this study is to investigate the effect of the hot weather conditions of UAE on the changes in thermal characteristics and the heat transfer of the embedded insulation material at different wall locations, considering the dynamic change of the thermal conductivity of the insulation.

\section{Methodology}

The research was run in two phases. The first phase considers the insulating material with low-density level and compare its performance when the analysis run applying both constant and variable thermal conductivity as a relationship of weather temperature. Variations in the thermal conductivity of the insulation were explored in relation to the operating temperature.

Although, the effect of changeable heat capacity seems to be bigger than the effect of variable thermal conductivity, the main focus of this research as mentioned is to study the pure effect of changeable ambient temperature on the thermal conductivity of the insulation material placed in different wall section positions while the other material thermal properties held constant.

Different positions of the insulating material were applied to select the optimum location to lower the heat transfer into the building through the wall assemble and consequently lower the energy consumption of applied HVAC system. The selected locations of insulating material defined by positions 1, 2 and 3 (as seen in Fig. 1), locating toward outside surface, toward inside surface and in the middle of wall section, respectively.

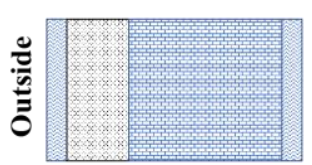

Position 1

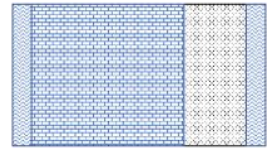

Position 2

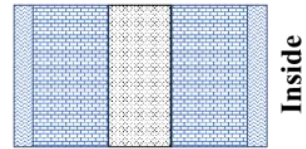

Position 3

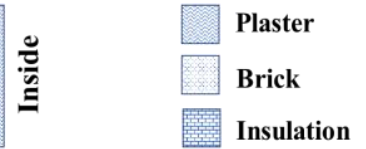

Insulation

Fig. 1: Position of the insulation material in the wall assemble.

After highlighting the importance of applying the variable thermal conductivity of the material, the research in the second part focuses in the changeable thermal conductivities of the insulating material considering different densities levels applied at different locations in the wall section.

For its supply, lower cost and wide use, expanded polystyrene was chosen for this analysis. The properties of the used insulation material is listed below in Table 1.

Table 1: Thermo-physical properties of the materials used in the experiments [6].

\begin{tabular}{|c|c|c|c|}
\hline \multirow{2}{*}{ Material } & Specific Heat Capacity & Density & Constant Thermal conductivity \\
\cline { 2 - 4 } & $(\mathrm{J} / \mathrm{kg} \mathrm{K})$ & $\left(\mathrm{kg} / \mathrm{m}^{3}\right)$ & $(\mathrm{W} / \mathrm{m} \mathrm{K})$ \\
\hline \multirow{3}{*}{$\begin{array}{c}\text { Expanded } \\
\text { Polystyrene }\end{array}$} & \multirow{3}{*}{1450} & 12 & $\lambda_{\text {LD,const }}=0.03936$ \\
\cline { 2 - 4 } & & 20 & $\lambda_{\text {HD,const }}=0.03714$ \\
\hline Plaster & 840 & 30 & $\lambda_{\text {UHD,const }}=0.0359$ \\
\hline Brick & 880 & 1700 & $\lambda_{\text {SHD,const }}=0.03444$ \\
\hline
\end{tabular}


The authors have shown in their previous researches, an inversely proportional linear relationship of $\lambda$-value which decreases with the increase in density of the sample [6]. Indeed, the impact of operating temperature on the thermal conductivity values of polystyrene insulation material with four density levels - low (LD), high (HD), ultra-high (UHD), and super-high (SHD) - is illustrated in Fig. 2. As can be seen from the graph, the thermal conductivity of all four samples is linear. While it is affected by the operating temperature to varying degrees, in all cases, higher temperature results in higher thermal conductivity. Moreover, thermal conductivity decreases with the increase in sample density.

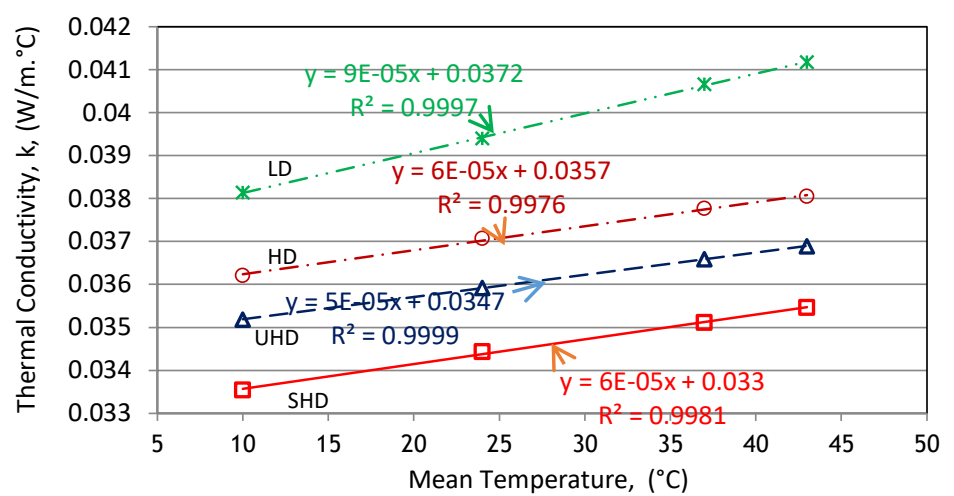

Fig. 2: Change of k-value with different densities vs. operating temperatures.

\subsection{Numerical Model}

For each position, a two-dimensional finite volume heat-transfer model of the building wall was built and, accordingly, the temperature distribution was analysed through the wall sections implementing the insulation's constant and variable thermal conductivity (see Fig. 3). Using the ANSYS platform adopting the hot environment state of Al Ain (UAE), the heat flow study through the wall segment was modelled and solved by applying the user-defined transient equations of weather conditions, and the variable thermal conductivity of the insulating material mentioned in Table 1 . The problem was solved temporarily (temperature and moisture). A mesh independent study was applied in the numerical model and the transient temperature distribution at each node was determined iteratively by a solver.

The thermal boundary conditions were applied in the numerical model as illustrated in Fig.4 and the temperature of the outer and inner surface was transiently monitored and the average surface temperature were used in post analysis.
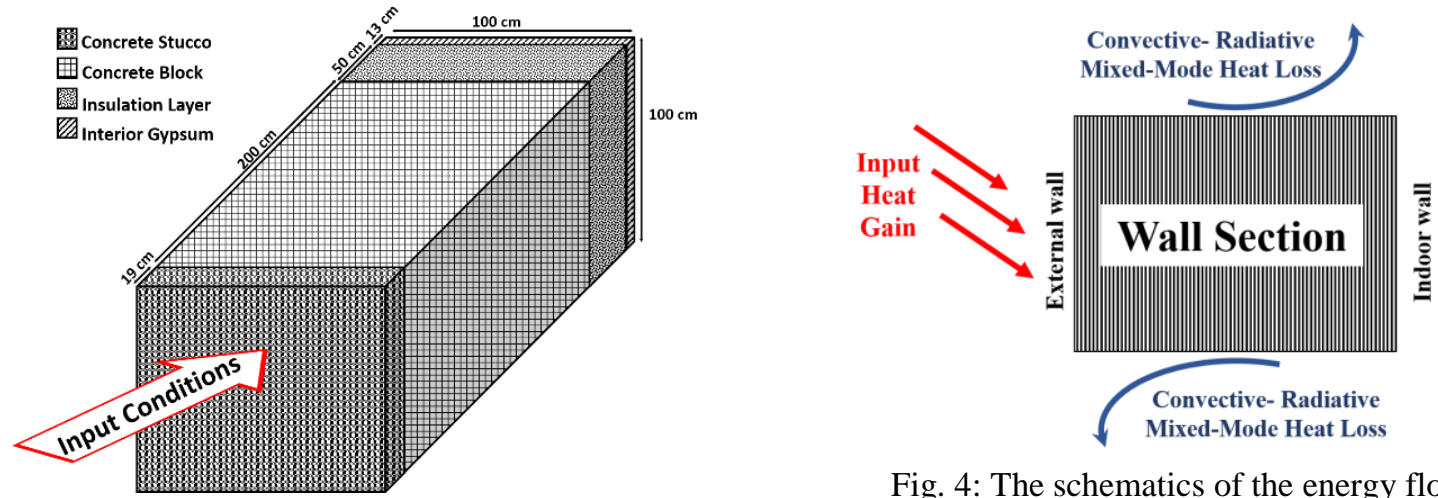

Fig. 3: Heat transfer analysis model

Fig. 4: The schematics of the energy flow in the wall section.

The exterior concrete stucco treated as the system input heat flux boundary (heat gain source), including incident irradiance $(q)$ with a convective-radiative mixed-mode heat loss. The indoor weather comfortable temperature was set to $25^{\circ} \mathrm{C}$. The other boundary conditions are treated as convective-radiative mixed-mode heat loss. 
The two-dimensional differential equation given below governs the transient heat transfer [20]:

$$
\rho c \frac{\partial T}{\partial t}-\left[\frac{\partial}{\partial x_{i}}\left(\lambda_{i j} \frac{\partial T}{\partial x_{j}}\right)\right]+Z_{c}+Z_{r}=0
$$

where $\rho$ denotes the density, $c$ is the heat capacity, $\lambda$ is the thermal conductivity of the material, $T$ represents the temperature, $t$ is the time, and $x_{i}$ and $x_{j}$ denote the unit vectors in x-direction and y-direction, respectively. The heat convection and radiation losses represented by $Z_{c}$ and $Z_{r}$ in Eq. (2) and Eq. (3) are calculated using the following expressions [21]:

$$
\begin{gathered}
Z_{c}=h_{c} A\left(T-T_{a m b}\right) \\
Z_{r}=\sigma \varepsilon A\left(T^{4}-T_{\infty}{ }^{4}\right)
\end{gathered}
$$

where $h_{c}$ is the convective heat loss coefficient, $A$ is the stucco wall surface area (0.92), $\sigma$ denotes the Stefan-Boltzman constant, $T_{a m b}$ is the ambient temperature, and $T_{\infty}$ represents the outdoor air temperature.

The thermal conductivity $(\lambda)$ of the insulation material is treated as a function of temperature and is defined by Eq. (4) [20]:

$$
\lambda(T)=C_{1}+C_{2} T+C_{2} T^{3} \ldots
$$

where $\lambda$ is the thermal conductivity of the material, $C_{i}$ are the constants and $T$ denotes the temperature.

\subsection{Numerical Validation}

Al Ain's (UAE) climate conditions were characterized by high solar radiation strength, high atmospheric temperature, and low-to-medium wind velocity, which were used as inputs for the numerical model. By comparing the simulated transient temperature with previous published experimental results reported by [22], the accuracy of the numerical model was evaluated. A concrete block was used in the experiment to validate the thermal model. The thickness of the concrete and the insulation layer was $2.5 \mathrm{~cm}$ and $1 \mathrm{~cm}$, respectively. A scaled down test chamber with dimensions of $40 \times 40 \times 40 \mathrm{~cm}^{3}$ was constructed behind the blocks to mimic an indoor space. All details of the experimentation are reported by [22]. Fig. 5 displays the evolution of the experimental and virtual transient temperature on the wall section's inner surface. A reasonable range (1 percent error) indicating the accuracy of the evolved thermal model was accepted between the experimental and simulated temperatures.

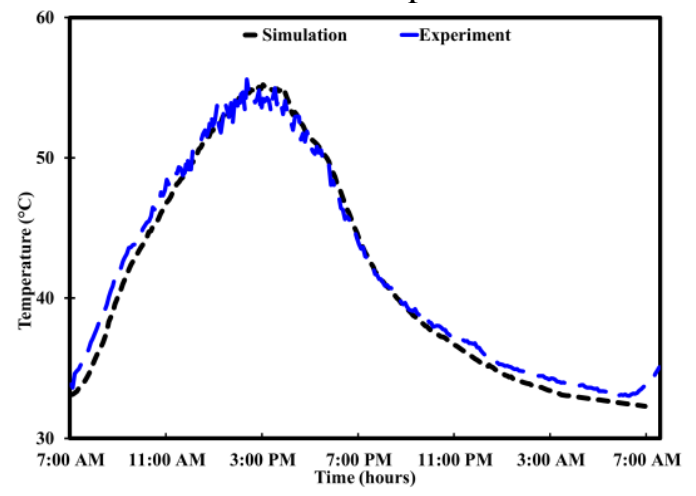

Fig. 5: The experimental and simulated transient temperature rise of the inner wall surface for a hot climatic weather of Al Ain, UAE.

\section{Result and Discussion}

The first part of this study was limited to the low density (LD) EPS. Both variable and constant thermal conductivities of the thermal insulating materials were compared. The temperature profiles at the outer and the inner surfaces of the wall sections integrating the insulation material at different positions are shown in Fig. 6 . The result shows that generally during the daytime, the use of a variable thermal conductivity model results in a higher increase in temperature on the inner surface compared to the constant thermal conductivity model of the insulation material on the three locations of the insulation inside the wall. This highlights the importance and the need of considering the variability in the thermal conductivity of the insulating material to obtain the correct sizing of HVAC system. The difference in the temperature rise between the constant and variable thermal conductivity models were analysed. The change in 
temperature $(\Delta T)$ between the variable and the constant thermal conductivity gradually increases as the ambient temperature and solar radiation rise.

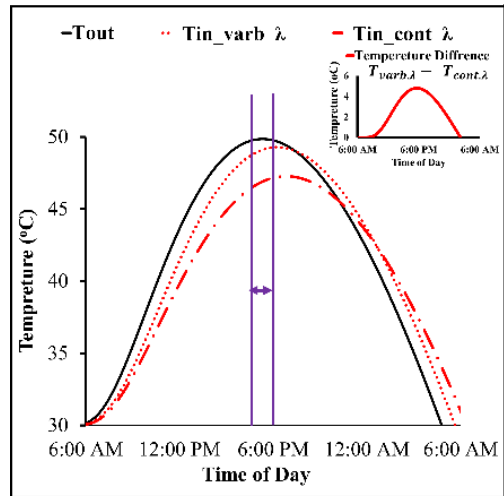

A

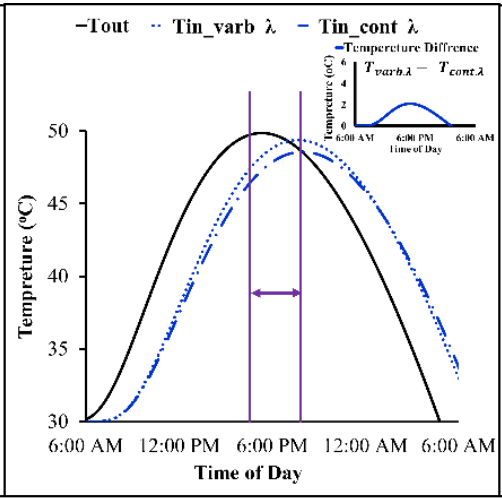

B

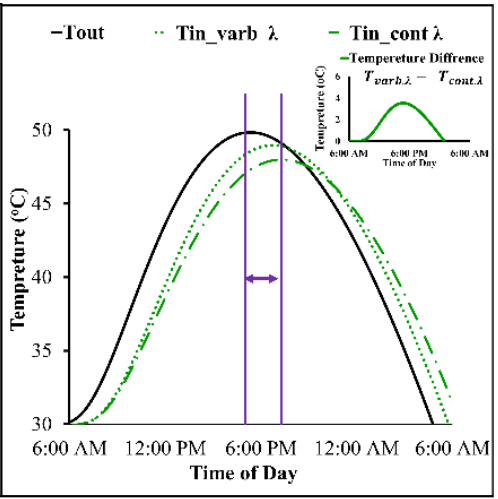

C

Fig. 6: Temperature profile of constant- $\lambda$ (solid lines) and variable- $\lambda$ (dashed lines) for LD polystyrene integrated in wall section in (A) Position 1, (B) Position 2, and (C) Position 3.

As shown in Fig.6, the temperature change $(\Delta \mathrm{T})$ between the constant and the variable $\lambda$-value of the EPS at the inner surface of the wall segment was measured at various insulation locations in the wall assembly. The maximum $\Delta \mathrm{T}$ are $4.7^{\circ} \mathrm{C}$, $2^{\circ} \mathrm{C}$ and $3.5^{\circ} \mathrm{C}$ at positions 1,2 , and 3, respectively. Hence, in conclusion, $\Delta \mathrm{T}$ decreases as the insulation material is located toward the inner surface due to heat loss within the wall layers. This point shows the necessary of considering the variable thermal conductivity of the insulating material to obtain appropriate sizing of the HVAC system especially if the insulating material is locating toward outside. This is due to the largest difference between the currently applied constant thermal conductivity performance of the material and the real case (changeable thermal conductivity performance) reaching up to $4.7^{\circ} \mathrm{C}$. While the difference was minimal if the insulating material is located toward inner surface.

To explain the obtained result, the dynamic thermal conductivity at various locations of the insulation material is shown in Fig. 7, thereby indicating that the thermal characteristic of the insulation material changes with the position of the insulation material. The dynamic thermal conductivity of the insulation material was found to be the highest $(0.0417 \mathrm{~W} / \mathrm{mK})$ for Position 1 due to the high surface temperature. For Position 2 and 3 of the insulation, the wall assembly provides better thermal resistance due to the heat loss through the different layers of the wall.

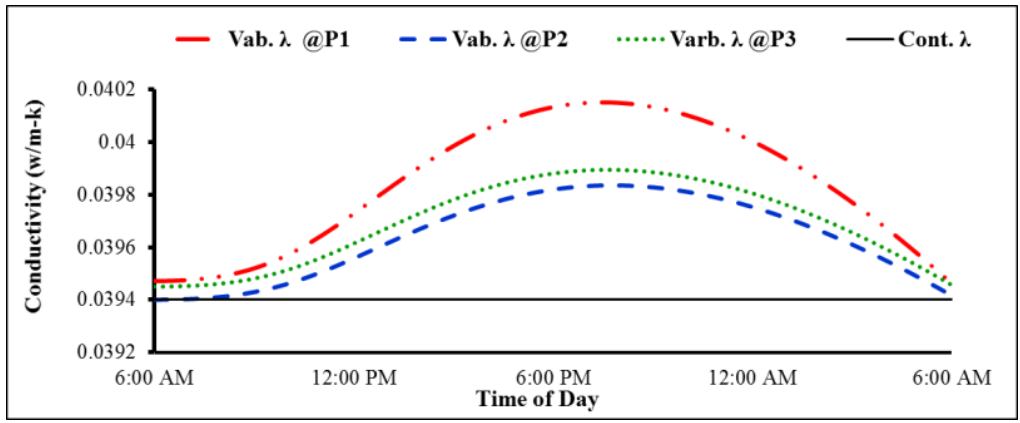

Fig. 7: Dynamic thermal conductivity (dashed lines) for LD polystyrene integrated in the three studied positions as compared to constant thermal conductivity.

These primary result reveal the importance of applying the variable thermal conductivity performance of the insulating material concluding that position 2 and 3 are indicating better performance. Another assessment criterion namely time lag was used to obtain the optimum location of the insulating material in the wall section. The time lag is the time difference between the maximum outer and inner wall surface temperatures considering the variable thermal conductivity of the 
insulating material. The time lag at different locations in the wall is presented also in Fig. 6 by the vertical solid lines. From the result, as the insulation material located toward the inner surface, the time lag between the outer and inner wall surfaces increase. The time lag where the insulation material is located toward the outer wall surface is around 2 hrs, while the time lag in case of locating the insulation material toward the inner surface is $4 \mathrm{hrs}$.

The net thermal effect (net temperature drop) applying the variable thermal conductivity of the insulation material on the heat transferred from the outer surface $\left(\mathrm{T}_{\text {out }}\right)$ of the building to the indoor space $\left(\mathrm{T}_{\text {in }}\right)$ is used as the final assessment criteria and compared to the reference case based on constant thermal conductivity, as shown in Fig. 8. It can be seen that the peak temperature of the inner surface of the wall section applying variable thermal conductivity (Fig. 8B) is generally higher than applying constant thermal conductivity (Fig. 8A) of the same insulation material. Thus, in case of applying constant thermal performance of the insulating material, a fault indication of the real amount of the heat transferred and consequently, the HVAC system is either underestimated or oversized.

Considering the dynamic aspect of the variable thermal conductivity of the insulation material as the research is highlighting, it is obvious that locating the thermal insulation material in the middle of the wall section (Position 3) generates a highest temperature drop during the day time while integrating the insulation material toward the outside shows the lowest temperature drop, thus concluding that the middle of the wall section is the optimum location to achieve the highest thermal resistance.

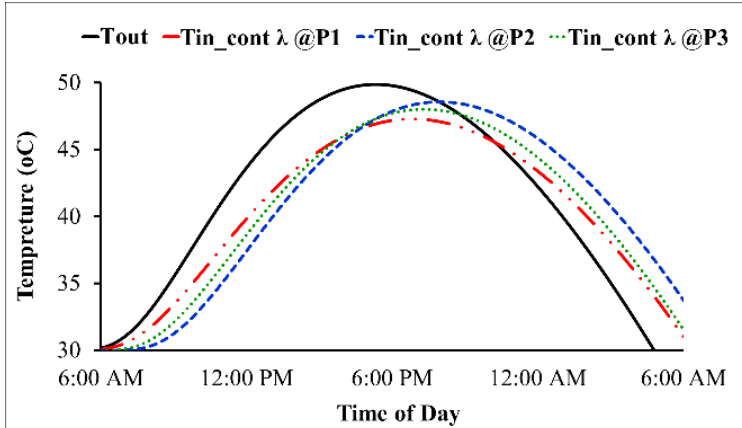

A

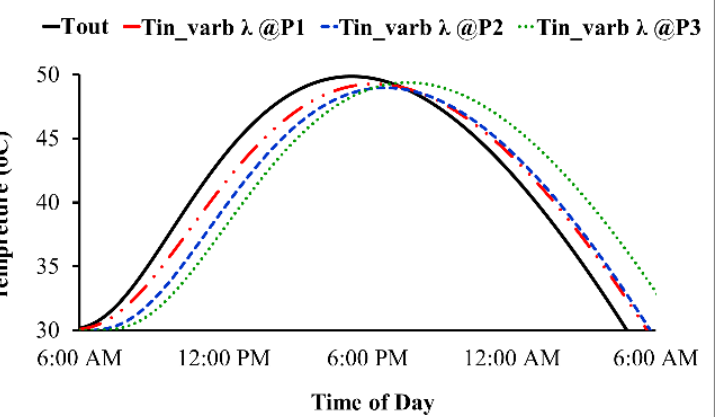

B

Fig. 8: Transient temperature evolution on the inner wall surface integrating the LD polystyrene at studied wall positions in case of (A) constant and (B) variable thermal conductivity of the polystyrene.

The percentage of the net heat reduction in cases of constant and variable thermal conductivity of the polystyrene located at three different positions in the wall assembly is shown in Fig. 9.

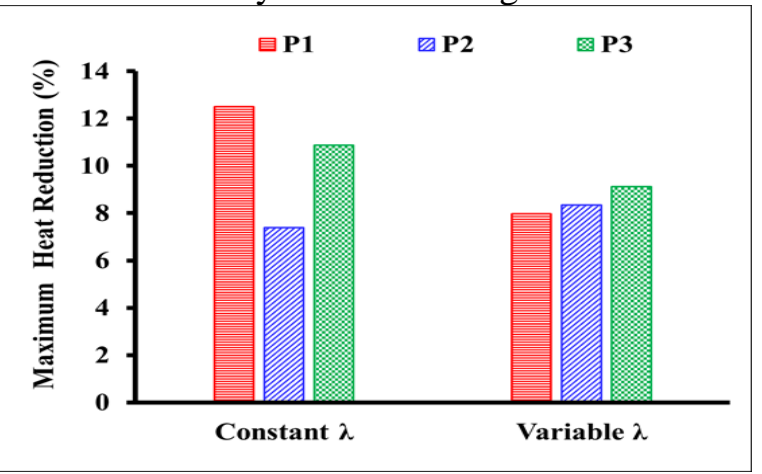

Fig.9: Percentage of the net heat reduction in case of constant and variable thermal conductivity of the LD polystyrene located at three different positions within the wall assembly.

From Fig. 9, it can be noted that applying a constant thermal conductivity of polystyrene shows that integrating the polystyrene toward the outer surface (Position 1) will provide the highest heat gain by conduction (up to 12.5\%), while integrating the polystyrene in positions 2 and 3 will provide a reduction percentage in heat gain by conduction of $7.3 \%$ 
and $10.8 \%$, respectively. However, in the case of applying variable thermal conductivity, the results are not in line with the previous ones. The highest percentage of the reduction in the heat gained by conduction is obtained for Position 3 with maximum reduction of $9.1 \%$.

This study has been extended to cover larger ranges of the density levels of the insulating material as mentioned previously in Table 1 focusing more in the variable thermal performance of the insulating material. The dynamic thermal conductivity of the four studied density levels of the EPS at different positions are illustrated in Fig. 10.

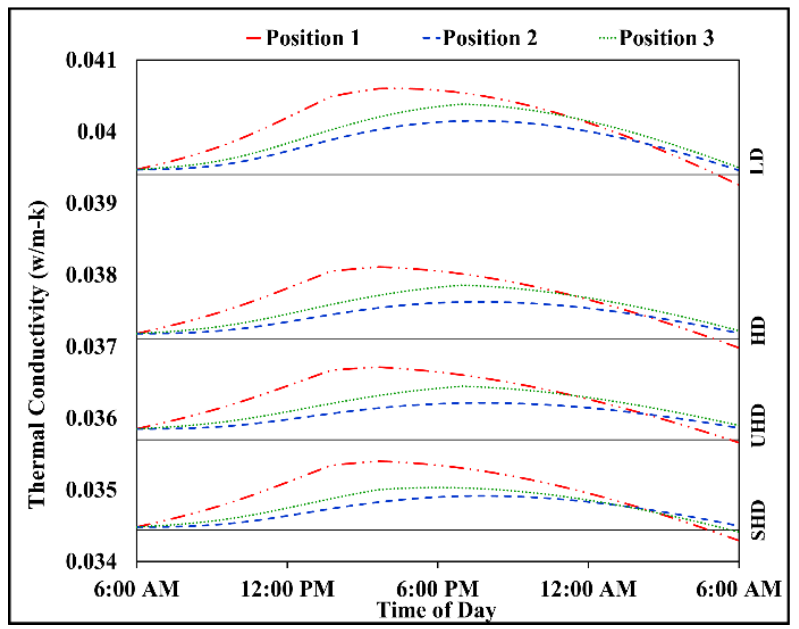

Fig. 10: Dynamic (dashed lines) and constant (solid lines) thermal conductivity of four density levels of EPS integrated in wall section in Position 1, 2 and 3.

Fig. 10 shows that the thermal conductivity of the four EPS density ranges varies with the insulation material's location. Starting with comparing the general profile of the thermal conductivity in term of daily temperature evolution at different positions in the wall section. Variable thermal conductivity performance at different positions show that the largest changes in the thermal conductivity is at Position 1 (where the insulation is located toward the outside wall surface) due to the high impact of the outside temperature as explained before in Fig. 7. Owing to the conductive heat loss across the wall layers, the change in the thermal conductivity is lower relative to the two other cases where the EPS is located toward the inner surface of the wall assembly (Positions 2 and 3). The same trend is observed for the four studied densities. This result confirmed the previous obtained results in phase 1 and emphasizes that the conclusion is valid at different density levels of insulating material.

\section{Conclusion}

The current work shows the dynamic effect of the EPS insulation variable thermal conductivity at various positions in the wall segment on the net reduction of heat through the wall assembly in the UAE's hot climate. Expanded polystyrene with four density ratios, adding the variable thermal conductivity ( $\lambda$-value) of polystyrene, was inserted into a standard wall building segment at various locations (outward, inward, and middle). A validated heat transfer model was used to account for the evolution of temperatures during the day on the inner wall surface.

The findings show that in the case of EPS located towards the outside surface for the four densities leads to the highest inner wall surface temperatures during daytime due to the highest dynamic change of the $\lambda$-value at this location. Locating the insulation layer at the core of the wall assembly results in the best thermal performance and the lowest inner wall surface temperature rise. However, the trend reverses at nighttime.

The change in temperature on the inner wall surface in case of applying the constant and variable $\lambda$-values decreases as the insulating material is located toward the inner wall surface of the building wall section. The findings indicate that the highest dynamic thermal efficiency is obtained by placing the insulation material in the centre of the wall assembly, taking into account the change in the thermal conductivity of the insulation. 


\section{Acknowledgements}

This work is supported by the Research Start-up grant, UAE University (Grant No. G00002665). The author would also to thank Eng. Shaimaa Abdelbaqi for the simulation results performed in this study.

\section{References}

[1] Briefing note 33: The Impacts of Construction and the Built Environment, Willmott Dixon Re-Thinking Ltd. (2010). URL: www.willmottdixon.co.uk/asset/download/9462.

[2] M. Lenzen, G.J. Treloar. Embodied energy in buildings: wood versus concrete-reply to Börjesson and Gustavsson, Energy Policy, 2002; 30:249-244.

[3] A.Z. Y1lmaz. Ak1llı Binalar ve Yenilenebilir Enerji (Smart Buildings and Renewable Energy), 7. Ulusal Tesisat Mühendisliği Konferans1, 23-26 Kasım 2005, İzmir, pp. 1-8.

[4] Lechner N, Heating, Cooling, Lighting Design Methods for Architects, Canada: John Wiley Sons; 1991.

[5] V. Olgyay. Design with Climate Bioclimatic Approach to Architectural Regionalism, Princeton, New Jersey: Princeton University Press; 1969.

[6] M. Khoukhi. The combined effect of heat and moisture transfer dependent thermal conductivity of polystyrene insulation material: Impact on building energy performance. Energy and Buildings 2018; 169:228-235.

[7] D.M.S. Al-Homoud. Performance characteristics and practical applications of common building thermal insulation materials. Building and Environment 2005; 40 (3):353-366.

[8] P. Gori, F. Bisegna. Thermophysical parameter estimation of multi-layer walls with stochastic optimization methods. International Journal of Heat and Technology 2010; 28:109- 116.

[9] U. Berardi. The impact of aging and environmental conditions on the effective thermal conductivity of several foam materials, Energy, 2019; 182, 777-794.

[10] U. Berardi. The impact of the temperature dependent thermal conductivity of insulating materials on the effective building envelope performance, Energy Procedia, 2017; 132, 237-242.

[11] K. Gradeci, U. Berardi, B. Time, J. Köhlerc. Evaluating highly insulated walls to withstand bio deterioration: a probabilistic-based methodology, Energy and Buildings, 2018; 177, 112-124,

[12] Berardi, U. A cross-country comparison of the building energy consumptions and their trends. Resources, Conservation and Recycling 2017; 123: 230-241.

[13] I. Budaiwi, A. Abdou, M. Al-Homoud. Variations of thermal conductivity of insulation materials under different operating temperatures: Impact on envelope-induced cooling load. Journal of Architectural Engineering 2002; 8(4):125132.

[14] M. Khoukhi, S. Abdelbaqi, A. Hassan. Yearly energy performance assessment of employing expanded polystyrene with variable temperature and moisture-thermal conductivity relationship. Materials 2019; 12(18):3000.

[15] M. Khoukhi, A. Hassan, S. Al Saadi, S. Abdelbaqi. A dynamic thermal response on thermal conductivity at different temperature and moisture levels of EPS insulation. Case Studies in Thermal Engineering, 2019; 14, 100481.

[16] M.C. Chyu, X. Zeng, L. Ye. The effect of moisture on the performance of polyurethane insulation used on a district heating and cooling pipe, ASHRAE Trans 1997; 309:317-103.

[17] M.C. Chyu, X. Zeng, L. Ye. Performance of fibers glass pipe insulation subjected to underground water attack, ASHRAE Trans 1997; 303; 308-103.

[18] M.C. Chyu, X. Zeng, L. Ye. Effect of underground water attack on the performance of mineral wool pipe insulation, ASHRAE Trans 1998; 168:175-104.

[19] X. Liu, C.H. Ge, P. Fazio, G. Chen, X. Guo. Determination of optimum insulation thickness of exterior walls with moisture transfer in hot summer and cold winter zone of China. Energy Building 2015; 361:368-109.

[20] I.A. Raja, J.F. Nicol, K.J. McCartney, M.A. Humphreys. Thermal comfort: Use of controls in naturally ventilated buildings. Energy Building, 2001, 33, 235-244.

[21] D. Zhou, C.Y. Zhao, Y. Tian. Review on thermal energy storage with phase change materials (PCMs) in building applications. Applied Energy, 2012, 92, 593-605.

[22] A. Hasan, K. Al-Sallal, H. Alnoman, Y. Rashid, S. Abdelbaqi. Effect of Phase Change Materials (PCMs) Integrated into a Concrete Block on Heat Gain Prevention in a Hot Climate. Sustainability 2016; 8 (10), 1009. 